\title{
An Improved Unscented Kalman Filter Algorithm for Radar Azimuth Mutation
}

\author{
Dazhang You, ${ }^{1}$ Pan Liu, ${ }^{1}$ Wei Shang $\left(\mathbb{D},{ }^{1}\right.$ Yepeng Zhang, ${ }^{1}$ Yawei Kang, ${ }^{1}$ and Jun Xiong ${ }^{2}$ \\ ${ }^{1}$ School of Mechanical Engineering, Hubei University of Technology, Wuhan, China \\ ${ }^{2}$ System Design Institute of Hubei Aerospace Technology Academy, Wuhan, China \\ Correspondence should be addressed to Wei Shang; bitshw@126.com
}

Received 15 April 2020; Revised 27 June 2020; Accepted 23 August 2020; Published 7 September 2020

Academic Editor: Jeremy Straub

Copyright (C) 2020 Dazhang You et al. This is an open access article distributed under the Creative Commons Attribution License, which permits unrestricted use, distribution, and reproduction in any medium, provided the original work is properly cited.

\begin{abstract}
An improved UKF (Unscented Kalman Filter) algorithm is proposed to solve the problem of radar azimuth mutation. Since the radar azimuth angle will restart to count after each revolution of the radar, and when the aircraft just passes the abrupt angle change, the radar observation measurement will have a sudden change, which has serious consequences and is solved by the proposed novel UKF based on SVD. In order to improve the tracking accuracy and stability of the radar tracking system further, the SVD-MUKF (Singular Value Decomposition-based Memory Unscented Kalman Filter) based on multiple memory fading is constructed. Furthermore, several simulation results show that the SVD-MUKF algorithm proposed in this paper is better than the SVD-UKF (Singular Value Decomposition of Unscented Kalman Filter) algorithm and classical UKF algorithm in accuracy and stability. Last but not the least, the SVD-MUKF can achieve stable tracking of targets even in the case of angle mutation.
\end{abstract}

\section{Introduction}

With the continuous development of flight technology, the speed maneuvering performance of aircraft is constantly improved, which poses a severe challenge to the detection and tracking performance of warning radar [1-3]. Radar has a long detection range, has high data accuracy, and is not affected by environmental factors such as illumination $[4,5]$. It is widely used in map location, obstacle detection, target recognition, and tracking. Besides, it is also an indispensable sensor for environmental perception. However, a radar also has some problems. At present, most radar algorithms only use the single-frame data environment perception obtained by a rotation of the radar. Due to the sparsity of radar data, this single-frame perception method has a low processing capacity for distant objects and fails to give play to radar's detection capability. In order to deal with the problem of sparse radar data, the method of increasing the number of radar scan lines is usually adopted $[6,7]$, but the radar azimuth angles will restart to count after each revolution of the radar, and the radar observation measurement will also occur when the aircraft just passes the sudden change in azimuth. Sudden changes will seriously affect the accuracy of the radar tracking system $[8,9]$.

In practice, the general method to deal with this problem is to convert the radar measurement data to the cartesian coordinate system $[10,11]$. In the spatial cartesian coordinate system, even if the azimuth of the target for the radar coordinate system is mutated, the coordinates of the $X, Y$, and $Z$ axes of the target also change continuously [12]. However, as a result of the error of radar measurement data, the direct use of coordinate transformation is biased, so the radar measurement data should be debiased. Liu and Wang [12-15] made an in-depth study on the unbiased measurement transformation problem and solved the problem well. However, in some cases, it is impossible to convert the radar measurement data into a cartesian coordinate system. For example, only by measuring azimuth and Doppler frequency shift can some radars accurately locate and track targets. In this respect, Subedi et al. and Guldogan et al. [16-18] did a lot of work and achieved good results. In addition, although some radars can simultaneously observe spatial position information and Doppler frequency shift, the Doppler frequency shift cannot be converted to a rectangular coordinate system. In these 
cases, the problem of azimuth mutation still exists, and the resulting measurement covariance singular matrix seriously affects the convergence of the tracking filter [19-21].

In the azimuth mutation, Hidayat et al. [22] used a positive definite matrix instead of the original singular matrix for local correction, which constituted the correction measurement. However, after the modified measurement, the whole filter will become a suboptimal filter, and the accuracy cannot be guaranteed. In this paper, instead of modifying the system itself, we proposed a filtering algorithm that can solve the problem of azimuth mutation.

The filtering methods of the nonlinear system mainly include EKF (extended Kalman filtering), UKF, and CKF (cubature Kalman filtering). EKF expands nonlinear functions into Taylor series and omits higher-order terms, which is generally applicable to weakly nonlinear systems. Compared with UKF and CKF, EKF has poor filtering performance under strong nonlinear condition, and the computation is large, which easily leads to filtering divergence [23]. The CKF algorithm proposed by Arasaratnam et al. $[24,25]$ is based on cubature transformation. This algorithm uses the spherical radial criterion to approximate the posterior mean value and covariance of states, obtains new state points through the system equation at $2 n$ cubature points with the same weight, and further deduces the predicted state points at the next moment. The whole process does not need to calculate the complex Jacobi matrix and has a good filtering effect. However, the main research on $\mathrm{CKF}$ is still theoretical and the engineering application is not mature enough at present. UKF uses UT transform to process the nonlinear transmission of mean and covariance, so its accuracy is higher than EKF [26]. Furthermore, UKF is widely used in target tracking, vehicle driving state estimation, navigation systems, and aircraft attitude estimation [27, 28]. Last but not the least, UKF overcomes the shortcomings that EKF is easy to diverge and has been fully applied in engineering practice. Therefore, in this paper, we focus on the UKF algorithm. Our main contribution to this paper is as follows:

(1) A modified radar measurement method is proposed, which converts the azimuth information about the radar measurement data into azimuth cosine and sine values, and solves the problem of sudden angle change caused by the target flight path crossing the radar coordinate quadrant

(2) An improved UKF algorithm based on SVD is proposed to solve the error singular matrix caused by the abrupt change of radar azimuth, which traditional UKF cannot handle. This is because the radar tracking system will produce the singular matrix which Cholesky decomposition cannot decompose in traditional UKF

(3) The multiple fading memory factor is proposed to further improve the accuracy of the radar tracking system. Different from the single fading memory factor $[29,30]$, the multiple fading memory factor does not break the symmetry of the original matrix struc-

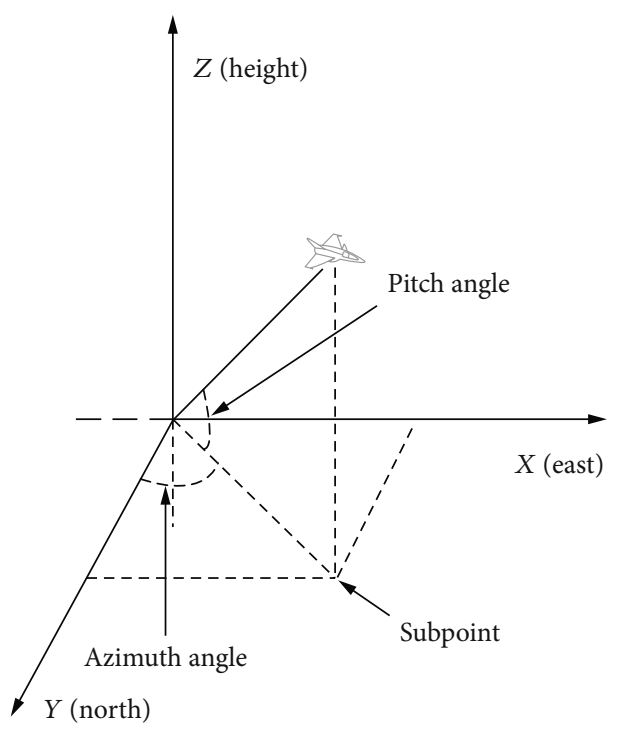

FIgURE 1: Radar tracking system ENU coordinate system.

turally, so that SVD decomposition can obtain the square root of the matrix stably, which improves the accuracy of the radar tracking system

Through simulation experiments, the SVD-MUKF algorithm and SVD-UKF algorithm were studied and compared; what is more, the applicability and stability of the algorithm in the radar tracking system were systematically analyzed. Therefore, we have drawn some conclusions on the indicators of the SVD-MUKF, providing a basis for solving the problem of radar tracking mutation angle.

The structure of this paper is as follows. On the basis of reviewing radar tracking theory, the model of a radar tracking system is firstly analyzed and modeled. In the solution section, the algorithm of the SVD-MUKF to solve the problem is studied in detail. At last, the simulation test is carried out and the calculation results are given.

\section{Theoretical Review}

In this section, the structural framework of the radar tracking system with modified measurement is given and the cause of a singular matrix is analyzed.

For radar tracking systems, the following discrete-time nonlinear systems are presented:

$$
\begin{gathered}
X(k+1)=\varphi X(k)+W(k), \\
Z(k)=h(X(k))+V(k),
\end{gathered}
$$

where $\varphi($.$) is the linear state transformation function, h$ (.) is the nonlinear measurement function, and $X \in R^{n}$ and $Z \in R^{m}$ are state vectors and observation vectors, respectively. All matrices have appropriate dimensions; $W(k)$ and $V(k)$ are zero-mean noncorrelated Gaussian random sequences.

In the radar tracking system, in the east-north-up (ENU) coordinate system (Figure 1), the value range of azimuth angle $\psi$ is generally $-\pi<\psi \leq \pi$. 

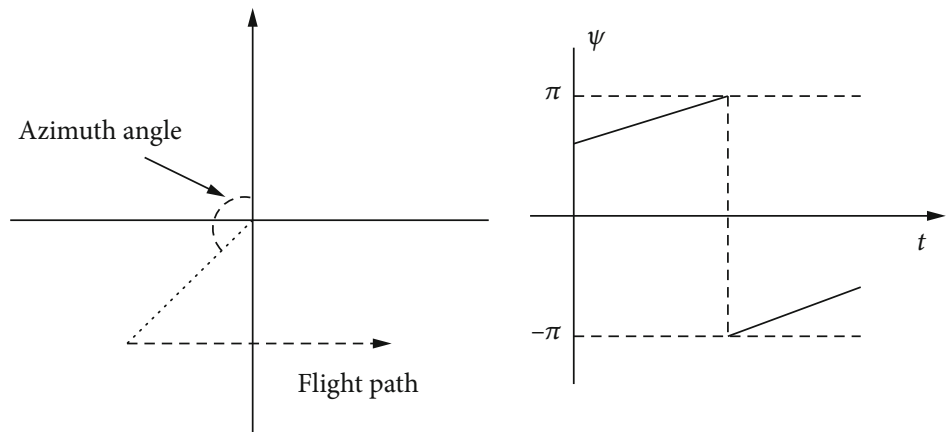

FIgure 2: Azimuth mutation.

If an aircraft flies from west to east and crosses the $Y O Z$ plane, then the observed value of radar will suddenly change from $\pi$ to $-\pi$, as shown in Figure 2 .

A sudden change in azimuth and the measured signal will cause the filter to diverge. We use the azimuth function here, where the value of the function changes continuously within the range of azimuth, and the filter can easily process the data. Here, we choose the cosine and sine of azimuth instead of azimuth information, and the observation vector is equivalent to

$$
\left[\rho \theta \cos (\psi) \sin (\psi) f_{d}\right]^{T}
$$

In the formula above, $\rho$ is the distance from the radar to the $\operatorname{target} ; \theta$ is the pitching angle; $f_{d}$ is the Doppler frequency shift.

We need to get the covariance matrix of the measurement in the UKF; let the measurement error of azimuth $\psi$ be equation reference goes here $\sigma_{\psi}^{2}$, the obtained azimuth value is converted into azimuth direction measurement.

$$
V_{\psi}=[\cos (\psi) \sin (\psi)]^{T} .
$$

Let the small $\psi$ be $\delta \psi$, we have a small change of $\delta V_{\psi}$.

$$
\delta V_{\psi}=\left[\begin{array}{ll}
-\sin (\psi) \delta \psi & \cos (\psi) \delta \psi
\end{array}\right]^{T}=\left[\begin{array}{ll}
-\sin (\psi) & \cos (\psi)
\end{array}\right]^{T} \delta \psi .
$$

If the small change quantity $\delta \psi$ represents the measurement error of azimuth angle $\psi$, the covariance matrix of $V_{\psi}$ can be obtained as

$$
\begin{aligned}
& R_{\psi}=E\left(\delta V_{\psi} \cdot \delta V_{\psi}^{T}\right)=E\left(\delta \psi^{2}\left[\begin{array}{c}
-\sin (\psi) \\
\cos (\psi)
\end{array}\right]\left[\begin{array}{ll}
-\sin (\psi) & \cos (\psi)]
\end{array}\right)\right. \\
& =\sigma_{\psi}^{2}\left[\begin{array}{cc}
\sin ^{2}(\psi) & -\sin (\psi) \cos (\psi) \\
-\sin (\psi) \cos (\psi) & \cos ^{2}(\psi)
\end{array}\right] .
\end{aligned}
$$

In the radar tracking system, the radar measurement is irrelevant, and the covariance matrix is a nonsingular matrix. Here, the azimuth information is converted to $V_{\psi}$, resulting in the local correlation of measurement, and $R_{\psi}$ is a singular matrix, which is represented by the covariance matrix

$$
\left|R_{\psi}\right|=\sigma_{\psi}^{2}\left|\begin{array}{cc}
\sin ^{2}(\psi) & -\sin (\psi) \cos (\psi) \\
-\sin (\psi) \cos (\psi) & \cos ^{2}(\psi)
\end{array}\right|=0
$$

In this case, the entire covariance matrix is

$$
R=\left[\begin{array}{ccccc}
\sigma_{P}^{2} & 0 & 0 & 0 & 0 \\
0 & \sigma_{\theta}^{2} & 0 & 0 & 0 \\
0 & 0 & \sigma_{\psi}^{2} \sin ^{2}(\psi) & -\sigma_{\psi}^{2} \sin (\psi) \cos (\psi) & 0 \\
0 & 0 & -\sigma_{\psi}^{2} \sin (\psi) \cos (\psi) & \sigma_{\psi}^{2} \cos ^{2}(\psi) & 0 \\
0 & 0 & 0 & 0 & \sigma_{f_{d}}^{2}
\end{array}\right] .
$$

$R$ must be singular in this case. The generated singular matrix contributes to the Cholesky decomposition to fail, which in turn causes the UKF algorithm to fail to track. Here, we improve the UKF algorithm and propose the SVD-MUKF algorithm.

\section{Singular Value Decomposition-Based Memory Unscented Kalman Filter}

3.1. Singular Value Decomposition Based on Unscented Kalman Filter. Unscented Kalman filter rejects the traditional method of linearization of nonlinear functions and adopts the Kalman filter framework. For the one-step prediction equation, the nonlinear transfer problem of mean and covariance is dealt with by UT (unscented transformation). The unscented Kalman filter algorithm approximates the probability density distribution of the nonlinear function and uses a series of samples to approximate the posterior probability density of the state.

The UKF process is as follows:

Nonlinear system:

$$
\left\{\begin{array}{l}
X_{k+1}=f\left(x_{k}\right)+W_{k} \\
Z_{k}=h\left(x_{k}\right)+V_{k}
\end{array}\right.
$$


$W(k)$ has the covariance matrix $Q ; V(k)$ has the covariance matrix $R$.

$$
\begin{aligned}
& X_{k, k}^{(i)}=\left[\widehat{X}_{k, k} \widehat{X}_{k, k}+\sqrt{(n+\lambda) P_{k, k}} \widehat{X}_{k, k}-\sqrt{(n+\lambda) P_{k, k}}\right], \\
& X_{k+1, k}^{(i)}=f\left[k, X_{k, k}^{(i)}\right] \\
& \widehat{X}_{k+1, k}=\sum_{i=0}^{2 n} \omega^{(i)} X_{k+1, k}^{(i)} \\
& P_{k+1, k}=\sum_{i=0}^{2 n} \omega^{(i)}\left[\widehat{X}_{k+1, k}-X_{k+1, k}^{(i)}\right]\left[X \wedge_{k+1, k}-X_{k+1, k}^{(i)}\right]^{T}+Q_{k} \text {, } \\
& X_{k+1, k}^{(i)}=\left[\widehat{X}_{k+1, k} \widehat{X}_{k+1, k}+\sqrt{(n+\lambda) P_{k+1 . k}} \widehat{X}_{k+1 . k}-\sqrt{(n+\lambda) P_{k+1 . k}}\right] \text {, } \\
& Z_{k+1, k}^{(i)}=h\left[X_{k+1, k}^{(i)}\right] \text {, } \\
& \bar{Z}_{k+1, k}=\sum_{i=0}^{2 n} \omega^{(i)} Z_{k+1, k}^{(i)} \\
& P_{z_{k} z_{k}}=\sum_{i=0}^{2 n} \omega^{(i)}\left[Z_{k+1, k}^{(i)}-\bar{Z}_{k+1, k}\right]\left[Z_{k+1, k}^{(i)}-\bar{Z}_{k+1, k}\right]^{T}+R, \\
& P_{x_{k} z_{k}}=\sum_{i=0}^{2 n} \omega^{(i)}\left[X_{k+1, k}^{(i)}-\bar{Z}_{k+1, k}\right]\left[Z_{k+1, k}^{(i)}-\bar{Z}_{k+1, k}\right]^{T} \\
& K_{k+1}=P_{X_{k} Z_{k}} P_{Z_{k} Z_{k}}^{-1} \\
& \widehat{X}_{k+1, k+1}=\widehat{X}_{k+1, k}+K_{k+1}\left[Z_{k+1}-\widehat{Z}_{k+1, k}\right] \text {, } \\
& P_{k+1, k+1}=P_{k+1, k}-K_{k+1} P_{z_{k} z_{k}} K_{k+1}^{T}
\end{aligned}
$$

where $n$ is the dimension of the state vector, $(\sqrt{P})^{T}(\sqrt{P}$ )$=P,(\sqrt{P})_{i}$ represents the $i$ th column of the square root of the matrix. Parameter $\lambda=\alpha^{2}(n+k)-n$ is a scaling factor. $\alpha$ is a constant that controls the distribution of the sample points. $k$ is a second-order scale parameter, usually set to 0 or $3-n$. $\beta$ is used to combine mean distribution with prior knowledge, and the weight of $\omega$ is given as follows:

$$
\left\{\begin{array}{l}
\omega_{m}^{(0)}=\frac{\lambda}{n+\lambda}, \\
\omega_{c}^{(0)}=\frac{\lambda}{n+\lambda}+\left(1-a^{2}+\beta\right), \\
\omega_{m}^{(i)}=\omega_{c}^{(i)}=\frac{\lambda}{2(n+\lambda)}, \quad i=1 \sim 2 n .
\end{array}\right.
$$

SVD is the most stable and accurate matrix decomposition algorithm in numerical algebra, which can be applied to the decomposition of any matrix. It represents a complex matrix by the product of several relatively simple matrices (eigenvalues and eigenvectors), which not only simplifies the operation but also preserves the characteristics of the original matrix.

There is a matrix one $U \in R^{m \times m}, \sum \in R^{m \times n}$, and $V \in R^{n \times n}$; the singular value of $A$ can be decomposed into

$$
A=U \sum V^{T}=U\left[\begin{array}{cc}
\sum & 0 \\
0 & 0
\end{array}\right] V^{T}
$$

where the singular value of $U \in R^{m \times m}, \sum \in R^{m \times n}, V \in R^{n \times n}$, $S=\operatorname{diag}\left(s_{1}, s_{2} \bullet \bullet s_{r}\right), s_{1}, s_{2} 2 \bullet \bullet s_{r}$ matrix $A, r$ is the rank of $B ; U$ and $V$ are the left and right singular vectors of $B$.

UT calculation in the UKF sigma needs to get the square root of the covariance matrix $P$ matrix. When the UKF random vector of the discussion of each component is independent of each other, namely, $P$ nonsingular matrix, we can use the Cholesky decomposition to calculate $P$ matrix square root, but some of the component or $P$ random vector is a singular matrix. In this case, we are unable to use Cholesky decomposition to get the square root of the matrix. In this paper, we use SVD to perform the singular value decomposition of covariance matrix $P$.

In view of the problem, we suppose that $P$ has a singular value decomposition:

$$
A=U \sum V^{T}=U\left[\begin{array}{ll}
\sum & 0 \\
0 & 0
\end{array}\right] V^{T}
$$

Since $P$ is the covariance matrix, we know that all $P$ matrices are paired $U=V ; P$ can be rewritten as

$$
P=U\left[\begin{array}{cc}
\sum & 0 \\
0 & 0
\end{array}\right] U^{H}
$$

If

$$
G=U\left[\begin{array}{cc}
\sum & 0 \\
0 & 0
\end{array}\right]^{1 / 2}=U\left[\begin{array}{cc}
\sum^{1 / 2} & 0 \\
0 & 0
\end{array}\right] P=U\left[\begin{array}{cc}
\sum & 0 \\
0 & 0
\end{array}\right] V^{H}
$$

And finally, we can get $P=G G^{H} ; G$ is the square root of the matrix $P$, computing $2 n+1$ sigma points:

$$
\begin{cases}X^{(0)}=\bar{X}, \quad i=0, & \\ X^{(i)}=\bar{X}+(\sqrt{(n+\lambda) G})_{i}, \quad i=1 \sim n, \\ X^{(i)}=\bar{X}-(\sqrt{(n+\lambda) G})_{i}, \quad i=n+1 \sim 2 n .\end{cases}
$$

SVD is used to solve the problem that Cholesky cannot use when the covariance matrix is singular.

3.2. Multiple Memory Fading Factor. In the UKF algorithm, the best memory forgetting factor can be obtained by the one-step algorithm of the best memory factor: 


$$
\lambda_{k}=\max \left\{1, \frac{1}{n} \operatorname{Tr}\left[N_{k} M_{k}^{-1}\right]\right\} .
$$

In the formula, the matrices 1 and 2 are, respectively,

$$
\begin{aligned}
& M_{k}=h\left(x_{k}\right) f\left(x_{k-1}\right) P_{k-1} f^{-1}\left(x_{k-1}\right) h^{-1}\left(x_{k}\right), \\
& N_{k}=C_{k}-h\left(x_{k}\right) Q_{k-1} h^{-1}\left(x_{k}\right)-R_{k} .
\end{aligned}
$$

$C_{k}$ is the error variance matrix; $Z_{k}$ is the estimated error:

$$
C_{k}=\frac{1}{k-1} \sum_{i=0}^{k} Z_{i} Z_{i}^{T}
$$

The optimal forgetting factor can be obtained from the following formula:

$$
\lambda_{k}=\max \left\{1, \frac{\operatorname{Tr}\left[N_{k}\right]}{\operatorname{Tr}\left[M_{k}\right]}\right\} .
$$

We can clearly see the physical significance of the optimal memory forgetting factor: the increase of estimation error $Z_{k}$ causes the increase of error variance matrix $C_{k}$. Furthermore, the optimum forgetting factor $\lambda_{k}$ increases correspondingly to make the filter reach the optimum. In the algorithm, the lower bound of the optimal forgetting factor $\lambda_{k}$ is defined as 1 , which is from the convergence consideration. It is generally considered that the actual filtering error is greater than the theoretical error.

In the field of surveying and mapping, the classical fading filter adjusts the state of the whole system with a single fading factor. In practice, it is often difficult to guarantee the best filter and describe the changes of the system state accurately. In order to solve this problem, a multifading filtering algorithm is proposed.

In multifading filtering, the prior covariance matrix of state parameters can be expressed as

$$
P_{k+1, k}=S_{k+1} \phi_{k+1, k} P_{k} \phi_{k+1, k}^{T}+Q_{k+1} \text {. }
$$

$S_{k+1}$ is the fading factor, and $S_{k+1}=\operatorname{diag}\left(s_{1}, s_{2}, \cdots \bullet s_{t}, 1,1\right.$, $\bullet \bullet 1)$. To ensure the symmetry of $P_{k+1, k}$, the left multiplication form of classical fading filter is replaced by the symmetric fading factor, which can avoid the harmful coupling of correlation caused by asymmetric fading factor.

$$
P_{k+1, k}=S_{k+1} \phi_{k+1, k} P_{k} \phi_{k+1, k}^{T} S_{k+1}^{T}+Q_{k+1} \text {. }
$$

Based on the one-step algorithm of optimal fading factor, we construct a new multimemory fading factor:

$$
S_{k+1}=\left\{\begin{array}{l}
\max \left\{1, \frac{\operatorname{tr}\left[N_{k}(i, i)\right]}{\operatorname{tr}\left[M_{k}(i, i)\right]}\right\}, \quad i=1,2, \cdots m, \\
1,
\end{array}\right.
$$

where $N_{k}(i, i)$ and $M_{k}(i, i)$ are the $i$ th diagonal elements of $N_{k}$ and $M_{k}$, respectively. Similarly, according to the

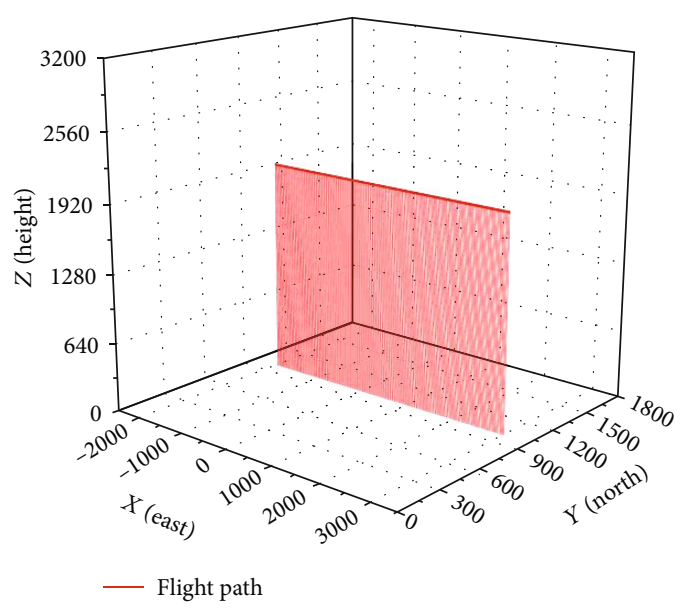

FIgURE 3: Trajectory of azimuth mutation (uniform).

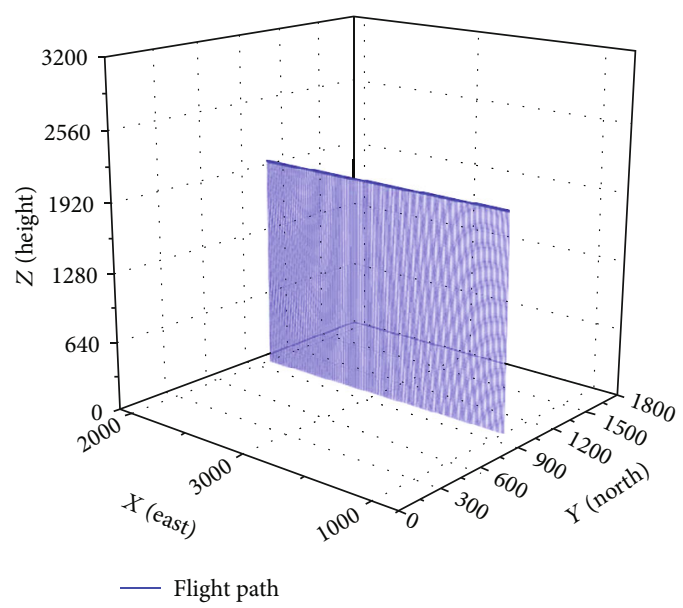

FIgURE 4: Trajectory without azimuth mutation (uniform).

observability of state parameters, only $m$ elements can be estimated adaptively, while the data of other diagonal elements are set to 1 . It can be seen that the new multiple memory fading factor constructed here has the following characteristics:

(1) The multiple fading factors are constructed on the basis of a single optimal fading factor, which ensures the optimum performance of the filter

(2) The memory factor can adjust the components of the prior state covariance matrix in real time, which can enhance the convergence performance of the filter and inhibit the divergence of the filter

(3) The calculation of multiple fading factors is simple and easy to realize

3.3. Singular Value Decomposition-Based Memory Unscented Kalman Filter. In the paper above, we replaced the Cholesky decomposition with SVD and performed UT to ensure that the error covariance singular matrix generated when the mutation angles that the radar generated would not affect the filtering. In the radar tracking system, a 9-dimensional 
TABLE 1: Uniform motion filter initialization conditions.

\begin{tabular}{|c|c|c|c|c|}
\hline Filter & Initialization state & Filtering covariance & System noise covariance & Measurement noise covariance \\
\hline UKF & $X_{0}^{U}$ & & & $R_{0}^{U}$ \\
\hline $\begin{array}{l}\text { SVD-UKF } \\
\text { SVD-MUKF }\end{array}$ & $X_{0}^{S}$ & $P_{0}$ & $Q_{0}$ & $R_{0}{ }^{S}$ \\
\hline
\end{tabular}

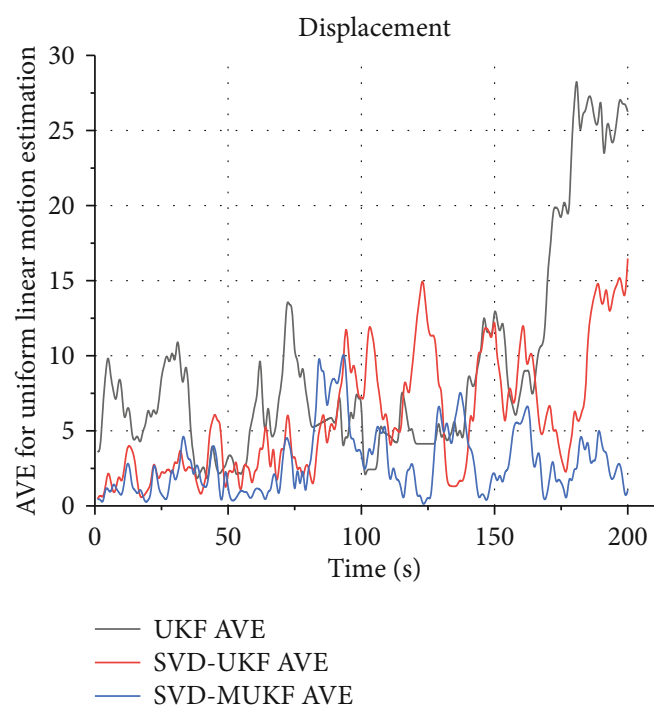

FIGURE 5: AVE for uniform linear motion estimation (displacement).

TABLE 2: Mean, variance, and RMSE of algorithm displacement data.

\begin{tabular}{lccc}
\hline Filter & AVE mean $(\mathrm{m})$ & AVE variance $(\mathrm{m})$ & RMSE $(\mathrm{m})$ \\
\hline UKF & 5.8910 & 4.1768 & 7.2216 \\
SVD-UKF & 9.1133 & 7.0373 & 11.5142 \\
SVD-MUKF & 2.8401 & 2.3075 & 3.6593 \\
\hline
\end{tabular}

state parameter vector is constructed, including carrier position, velocity, acceleration, and error in three directions. Under the framework of UKF, the iterative calculation formula for SVD-MUKF algorithm time constructed can be obtained as follows:

Time to update:

$$
\begin{aligned}
\widehat{X}_{k+1, k} & =\sum_{i=0}^{2 n} \omega^{(i)} X_{k+1, k}^{(i)}, \\
P_{k+1, k} & =S_{k}\left(\sum_{i=0}^{2 n} \omega^{(i)}\left[\widehat{X}_{k+1, k}-X_{k+1, k}^{(i)}\right]\left[X \wedge_{k+1, k}-X_{k+1, k}^{(i)}\right]^{T}\right) S_{k}^{T}+Q_{k} .
\end{aligned}
$$

In the radar tracking system, the observable variables are the distance from the aircraft to the radar, two extended azimuth angles, and pitch angles. Therefore, the dimension of

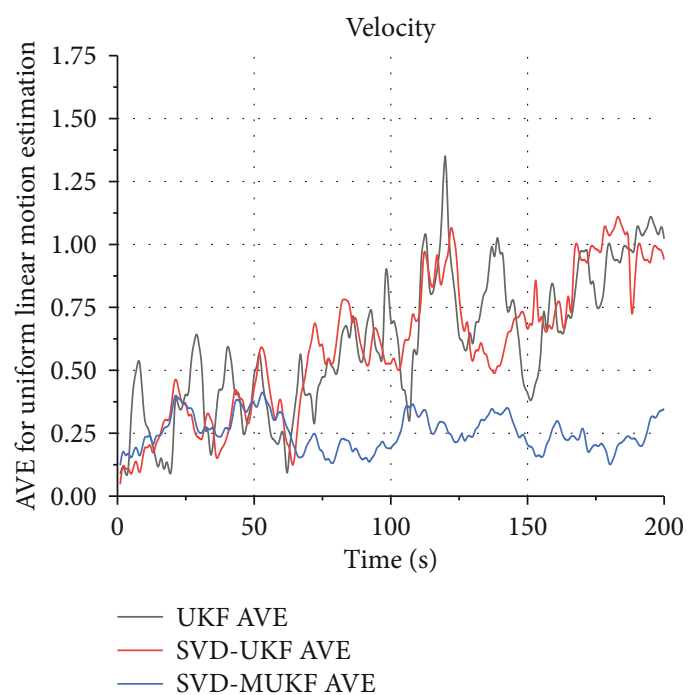

FIgURE 6: AVE for uniform linear motion estimation (velocity).

the observation equation is 5 , which can be estimated adaptively, and no element is set as 1 .

\section{Simulation}

The performance of the SVD-UKF, SVD-MUKF, and traditional UKF algorithm was comprehensively compared through simulation experiments. The traditional UKF algorithm could not run steadily after the mutation angle, so we adopted the same path but different starting points to prevent the trajectory from passing the mutation angle. By analyzing the results, the effectiveness of the SVD-MUKF algorithm is verified.

The error estimation in the object chart is obtained by AVE (absolute value error) and RMSE (root mean square error), which can be expressed as

$$
\begin{aligned}
\operatorname{AVE}(k) & =\sqrt{\left(X_{k}-X \wedge_{k}^{m}\right)^{2}}, \\
\operatorname{RMSE} & =\sqrt{\frac{1}{N} \sum_{i=1}^{N}\left(X_{k}-X \wedge_{k}^{m}\right)^{2}},
\end{aligned}
$$

where $X_{k}$ is the real value, $\widehat{X}_{k}^{m}$ is the predicted value, and $N$ is the number of steps in the total simulation time, that is, the total time.

For the purpose of testing the performance of three algorithms, AVE for displacement and speed and RMSE based on three algorithms are compared in the case of uniform speed 
motion. Besides, under the circumstances of nonuniform speed motion, we compare AVE of displacement and speed and RMSE of algorithms to verify the efficiency of the improved algorithm.

4.1. Simulation of Uniform Motion. A uniform line is defined as

(1) The UAV goes through azimuth mutation, as shown in Figure 3

(2) The UAV does not undergo azimuth mutation, as shown in Figure 4

The initialization bar is shown in Table 1.

The state is determined by a 9-dimensional vector including displacement, velocity, and acceleration:

$$
\begin{aligned}
& X_{0}^{U}=[-2000 ; 2000 ; 5000 ; 20 ; 0 ; 0 ; 0 ; 0 ; 0]^{T}, \\
& X_{0}^{S}=[2000 ; 2000 ; 5000 ; 20 ; 0 ; 0 ; 0 ; 0 ; 0]^{T}, \\
& P_{0}=\operatorname{diag}\left([10 ; 10 ; 10 ; 1 ; 1 ; 1 ; 0.1 ; 0.1 ; 0.1]^{2}\right)^{T}, \\
& Q_{0}=\operatorname{diag}\left([1 ; 1 ; 1 ; 0.1 ; 0.1 ; 0.1 ; 0.01 ; 0.01 ; 0.01]^{2}\right)^{T}, \\
& R_{O}^{U}=\operatorname{diag}\left([60 ; 0.01 ; 0.01 ; 0.01 ; 2]^{2}\right)^{T},
\end{aligned}
$$$$
R_{0}{ }^{S}=\left[\begin{array}{ccccc}
60^{2} & 0 & 0 & 0 & 0 \\
0 & 0.01^{2} & 0 & 0 & 0 \\
0 & 0 & 0.01^{2} & -0.01^{2} & 0 \\
0 & 0 & -0.01^{2} & 0.01^{2} & 0 \\
0 & 0 & 0 & 0 & 2^{2}
\end{array}\right] .
$$

In the radar system with position as the measurement object, the estimation performance of displacement by the three methods is shown in Figure 5 and Table 2. In the radar system with velocity as the measurement object, the estimation performance of velocity by the three methods is shown in Figure 6 and Table 3.

In Figures 5 and 6, the AVE values of UKF, SVD-UKF, and SVD-MUKF are given as time changes and the variation law of AVE values is given. In Tables 2 and 3, the data analysis values of displacement and velocity filtering results of three kinds of filters are given, and the filtering effect is more intuitive.

4.2. Simulation of Uniform Variable Motion. A uniform line is defined as

(1) The UAV performs a large maneuver with a 180degree turn and a sudden change in azimuth, as shown in Figure 7

(2) The UAV does not follow the same trajectory through the abrupt azimuth, as shown in Figure 8
TABLE 3: Mean, variance, and RMSE of algorithm velocity data.

\begin{tabular}{lccc}
\hline Filter & AVE mean $(\mathrm{m} / \mathrm{s})$ & $\begin{array}{c}\text { AVE variance } \\
(\mathrm{m} / \mathrm{s})\end{array}$ & $\begin{array}{c}\text { RMSE } \\
(\mathrm{m} / \mathrm{s})\end{array}$ \\
\hline UKF & 0.5966 & 0.2774 & 0.6580 \\
SVD-UKF & 0.6080 & 0.2857 & 0.6718 \\
SVD- & 0.2525 & 0.0706 & 0.2622 \\
MUKF & & & \\
\hline
\end{tabular}

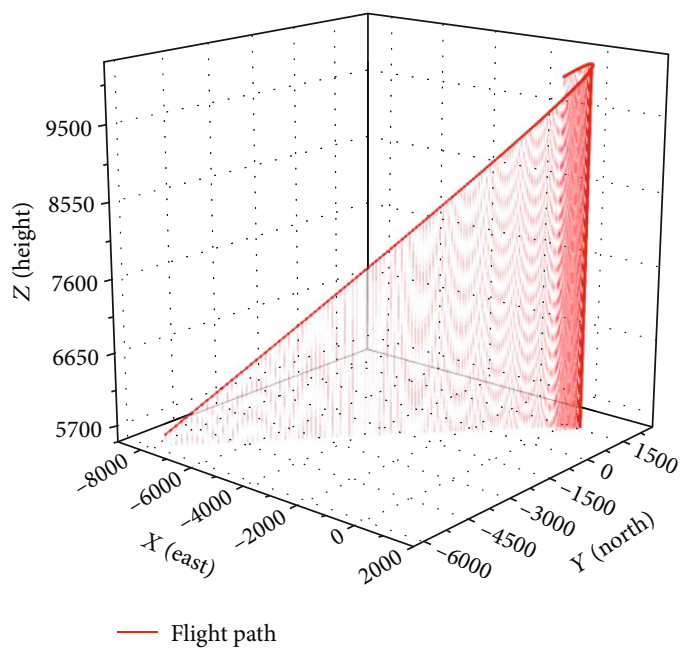

FIGURE 7: Trajectory of azimuth mutation (uniform variable).

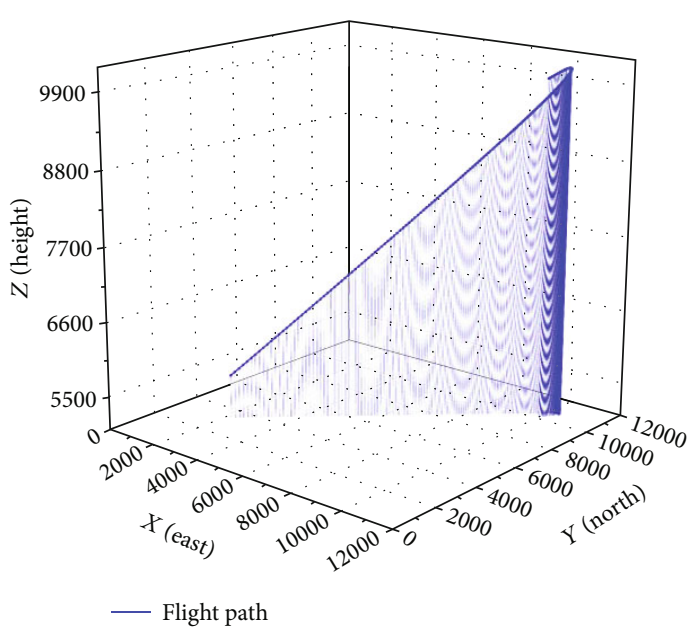

FIGURE 8: Trajectory without azimuth mutation (uniform variable).

TABLE 4: Uniform variable motion filter initialization conditions.

\begin{tabular}{lcccc}
\hline Filter & $\begin{array}{c}\text { Initialization } \\
\text { state }\end{array}$ & $\begin{array}{c}\text { Filtering } \\
\text { covariance }\end{array}$ & $\begin{array}{c}\text { System } \\
\text { noise } \\
\text { covariance }\end{array}$ & $\begin{array}{c}\text { Measurement } \\
\text { noise } \\
\text { covariance }\end{array}$ \\
\hline UKF & $X_{0}^{U}$ & & & $R_{0}{ }^{U}$ \\
SVD- & & $P_{0}$ & $Q_{0}$ & \\
UKF & $X_{0}^{S}$ & & & $R_{0}{ }^{S}$ \\
SVD- & & & & \\
MUKF & & & & \\
\hline
\end{tabular}




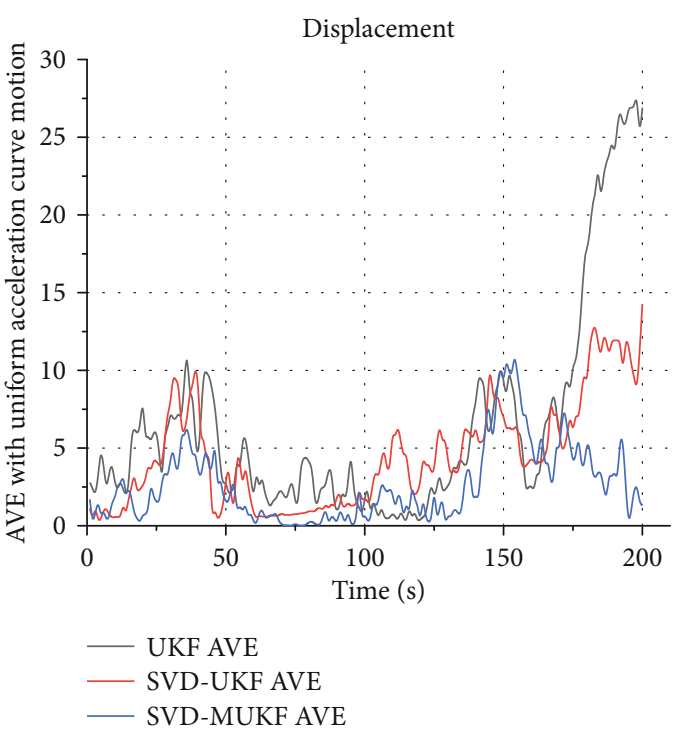

FIgURE 9: AVE with uniform acceleration curve motion (displacement).

TABLE 5: Mean, variance, and RMSE of algorithm displacement data.

\begin{tabular}{lccc}
\hline Filter & AVE mean $(\mathrm{m})$ & AVE variance $(\mathrm{m})$ & RMSE $(\mathrm{m})$ \\
\hline UKF & 4.0169 & 3.4446 & 5.2911 \\
SVD-UKF & 7.4619 & 6.7845 & 9.3695 \\
SVD-MUKF & 2.4578 & 2.4573 & 3.4713 \\
\hline
\end{tabular}

The initialization bar is shown in Table 4 .

$$
\begin{aligned}
& X_{0}^{U}=[-50 ; 1000 ; 10000 ; 50 ; 60 ; 20 ;-10 ;-10 ;-5]^{T}, \\
& X_{0}^{U}=[10000 ; 10000 ; 10000 ; 50 ; 60 ;-20 ;-10 ;-10 ;-5]^{T} .
\end{aligned}
$$

$P_{0} Q_{0} R_{O}^{U} R_{0}^{S}$ are the same as constant motion.

In a radar system for measuring position, three methods of displacement estimation performance are shown in Figure 9 and shown in Table 5, the measurement for speed of a radar system; the three methods of speed estimation performance are shown in Figure 10 and Table 6, based on the acceleration measurement of a radar system; the performance of the three methods of acceleration estimation is shown in Figure 11 and Table 7.

Figures 9 and 10 are similar to the uniform velocity motion and indicate the change of AVE with time. Based on the displacement and velocity, we increased the acceleration in Figure 11. AVE better reflects the filtering conditions of the three filters in uniform acceleration. The statistical results of the three types of filtered displacement, velocity, and acceleration data are given in Tables 5-7, respectively.

4.3. The Simulation Results. By comparing the AVE and RMSE of the three filtering algorithms, the conclusion can be drawn as follows:

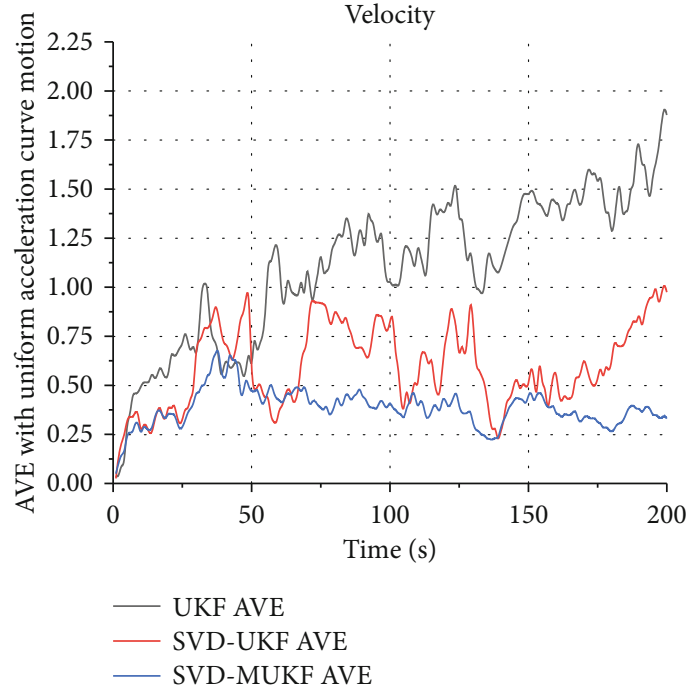

FIGURE 10: AVE with uniform acceleration curve motion (velocity).

TABLE 6: Mean, variance, and RMSE of algorithm velocity data.

\begin{tabular}{lccc}
\hline Filter & AVE mean $(\mathrm{m} / \mathrm{s})$ & $\begin{array}{c}\text { AVE variance } \\
(\mathrm{m} / \mathrm{s})\end{array}$ & $\begin{array}{c}\text { RMSE } \\
(\mathrm{m} / \mathrm{s})\end{array}$ \\
\hline UKF & 0.6006 & 0.2153 & 0.6371 \\
SVD-UKF & 1.1023 & 0.3774 & 1.1651 \\
SVD- & 0.3739 & 0.0765 & 0.3817 \\
MUKF & & & \\
\hline
\end{tabular}

(1) The ordinary SVD-UKF algorithm is slightly better than the traditional UKF algorithm in accuracy. This is because SVD has better stability than Cholesky decomposition, reducing the error generated by the system during the decomposition process

(2) The performance of the SVD-MUKF is better than that of the UKF and SVD-UKF in both displacement, velocity, and acceleration estimations. This is because the SVD-MUKF increases the memory fading factor on the basis of the SVD-UKF, eliminating the realtime adjustment of the test state covariance matrix by the UKF through the size of new information and making it expand the corresponding multiple, thus reducing the use efficiency of historical data. It can be seen from Figures 5, 6, and 9-11 that SVDMUKF's data processing results are significantly better than other filtering results; from Tables 2, 3, and 5-7, the AVE, variance, and RMSE values of displacement, velocity, and acceleration of the SVD-MUKF algorithm are far less than those of the other two methods, which illustrate the fading memory factor on the filter for the length of the memory of a certain limitation and improve the efficiency of the usage of new measurement information. The filtering divergence has played a certain inhibition

(3) Due to the large steering in the acceleration simulation of the aircraft, from Figure 7 to Figure 9, we 


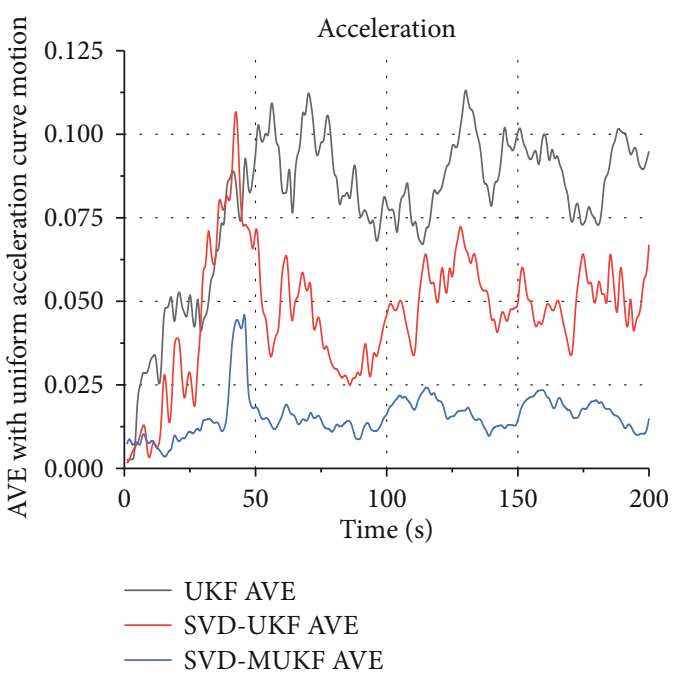

Figure 11: AVE with uniform acceleration curve motion (acceleration).

TABLE 7: Mean, variance, and RMSE of algorithm acceleration data.

\begin{tabular}{lccc}
\hline Filter & $\begin{array}{c}\text { AVE mean } \\
\left(\mathrm{m} / \mathrm{s}^{2}\right)\end{array}$ & $\begin{array}{c}\text { AVE variance } \\
\left(\mathrm{m} / \mathrm{s}^{2}\right)\end{array}$ & $\begin{array}{c}\text { RMSE } \\
\left(\mathrm{m} / \mathrm{s}^{2}\right)\end{array}$ \\
\hline UKF & 0.044 & 0.019 & 0.0476 \\
SVD-UKF & 0.081 & 0.023 & 0.0795 \\
$\begin{array}{l}\text { SVD- } \\
\text { MUKF }\end{array}$ & 0.015 & 0.005 & 0.0157 \\
\hline
\end{tabular}

can see that in the first 50 seconds, the errors of the three filters will increase sharply, but after the end of the steering, the errors will gradually return to the normal state, indicating that the above algorithm is not strong in tracking the sudden displacement. From 50 seconds to 150 seconds, the displacement gradually becomes stable, and there is no jump in the errors of displacement, velocity, and acceleration, which again confirms the above conclusion

\section{Conclusion}

In this paper, an effective solution, namely, the SVDMUKF tracking algorithm, is proposed to solve the azimuth mutation problem caused by the coordinate system selection problem of observation radar in a specific scene. Combined with the research focus of the UKF algorithm, the simulation data of the SVD-MUKF algorithm was compared with those of the SVD-UKF algorithm and SVDMUKF algorithm. Experimental results show that the SVD-MUKF tracking algorithm can not only solve the problem of angle mutation but also improve the accuracy of the SVD-MUKF algorithm compared with the SVDUKF algorithm and UKF algorithm.

\section{Data Availability}

The data used to support the findings of this study have not been made available because the National Natural Science Foundation of China (No. 51875180) has not yet completed its project.

\section{Conflicts of Interest}

The authors declare that there is no conflict of interest regarding the publication of this article.

\section{Acknowledgments}

This work was financed by a grant-in-aid for scientific research from the National Natural Science Foundation of China (No. 51875180).

\section{References}

[1] J. Yan, W. Pu, S. Zhou, H. Liu, and Z. Bao, "Collaborative detection and power allocation framework for target tracking in multiple radar system," Information Fusion, vol. 55, pp. 173-183, 2020.

[2] P. You, Z. Ding, L. Qian et al., "A motion parameter estimation method for radar maneuvering target in Gaussian clutter," IEEE Transactions on Signal Processing, vol. 67, no. 20, pp. 5433-5446, 2019.

[3] Z. Na'e, S. Yang, S. Xiyu, and C. Song, "Joint resource allocation scheme for target tracking in distributed MIMO radar systems," Journal of Systems Engineering and Electronics, vol. 30, no. 4, pp. 709-719, 2019.

[4] J. W. Paik and J. H. Lee, "Improvement of DOD/DOA estimation for bistatic MIMO radar," International Journal of Aeronautical and Space Sciences, vol. 20, no. 4, pp. 997-1009, 2019.

[5] X. Chen, D. Li, X. Yang, and H. Li, "Radar emitter signals identification with a optimal recurrent type 2 wavelet fuzzy neural network," International Journal of Aeronautical and Space Sciences, vol. 19, no. 3, pp. 685-693, 2018.

[6] P. Checchin, F. Gérossier, C. Blanc, R. Chapuis, and L. Trassoudaine, "Radar scan matching SLAM using the Fourier-Mellin transform," in Field and Service Robotics. Springer Tracts in Advanced Robotics, vol 62Springer, Berlin, Heidelberg.

[7] M. Grecu and W. F. Krajewski, "An efficient methodology for detection of anomalous propagation echoes in radar reflectivity data using neural networks," Journal of Atmospheric \& Oceanic Technology, vol. 17, no. 2, pp. 121-129, 2000.

[8] P. Bahl and V. N. Padmanabhan, "RADAR: an in-building RFbased user location and tracking system," in Proceedings IEEE INFOCOM 2000. Conference on Computer Communications. Nineteenth Annual Joint Conference of the IEEE Computer and Communications Societies (Cat. No.00CH37064), vol. 2, Tel Aviv, Israel, 2000.

[9] A. De Maio, S. De Nicola, Y. Huang, S. Zhang, and A. Farina, "Code design to optimize radar detection performance under accuracy and similarity constraints," IEEE Transactions on Signal Processing, vol. 56, no. 11, pp. 5618-5629, 2008.

[10] W. C. Chew and W. H. Weedon, "A 3D perfectly matched medium from modified Maxwell's equations with stretched 
coordinates," Microwave and Optical Technology Letters, vol. 7, no. 13, pp. 599-604, 1994.

[11] Y. Hao, Z. Xiong, F. Sun, and X. Wang, "Comparison of unscented Kalman filters," in 2007 International Conference on Mechatronics and Automation, pp. 895-899, Harbin, 2007.

[12] B. Liu and Z. Wang, "Improved calibration method for depolarization lidar measurement," Optics Express, vol. 21, no. 12, pp. 14583-14590, 2013.

[13] S. Bordonaro, P. Willett, and Y. Bar-Shalom, "Decorrelated unbiased converted measurement Kalman filter," IEEE Transactions on Aerospace and Electronic Systems, vol. 50, no. 2, pp. 1431-1444, 2014.

[14] B. D. Kim and J. S. Lee, "Decoupled tracking filter with modified unbiased converted measurements," in 2006 CIE International Conference on Radar, pp. 1-4, Shanghai, China, 2006.

[15] A. M. Manceur and P. Dutilleul, "Unbiased modified likelihood ratio tests for simple and double separability of a variance-covariance structure," Statistics \& Probability Letters, vol. 83, no. 2, pp. 631-636, 2013.

[16] S. Subedi, Y. D. Zhang, M. G. Amin, and B. Himed, "Group sparsity based multi-target tracking in passive multi-static radar systems using Doppler-only measurements," IEEE Transactions on Signal Processing, vol. 64, no. 14, pp. 36193634, 2016.

[17] M. B. Guldogan, D. Lindgren, F. Gustafsson, H. Habberstad, and U. Orguner, "Multi-target tracking with PHD filter using Doppler-only measurements," Digital Signal Processing, vol. 27, pp. 1-11, 2014

[18] J. H. Yoon, D. Y. Kim, S. H. Bae, and V. Shin, "Joint initialization and tracking of multiple moving objects using Doppler information," IEEE Transactions on Signal Processing, vol. 59, no. 7, pp. 3447-3452, 2011.

[19] S. H. Lee and M. West, "Convergence of the Markov chain distributed particle filter (MCDPF)," IEEE Transactions on Signal Processing, vol. 61, no. 4, pp. 801-812, 2013.

[20] B. Sinopoli, L. Schenato, M. Franceschetti, K. Poolla, M. I. Jordan, and S. S. Sastry, "Kalman filtering with intermittent observations," IEEE Transactions on Automatic Control, vol. 49, no. 9, pp. 1453-1464, 2004.

[21] R. Chen and J. S. Liu, "Mixture Kalman filters," Journal of the Royal Statistical Society: Series B (Statistical Methodology), vol. 62, no. 3, pp. 493-508, 2000.

[22] R. Hidayat, I. N. Rifai, W. Widada, and A. P. Adi, "Doppler circular array antenna principle for determining azimuth angle of radio transmitter," in 2011 International Symposium on Intelligent Signal Processing and Communications Systems (ISPACS), pp. 1-4, Chiang Mai, 2011.

[23] S. J. Julier and J. K. Uhlmann, "Unscented filtering and nonlinear estimation," Proceedings of the IEEE, vol. 92, no. 3, pp. 401422, 2004.

[24] I. Arasaratnam and S. Haykin, "Cubature Kalman filters," IEEE Transactions on Automatic Control, vol. 54, no. 6, pp. 1254-1269, 2009.

[25] B. Jia, M. Xin, and Y. Cheng, "High-degree cubature Kalman filter," Automatica, vol. 49, no. 2, pp. 510-518, 2013.

[26] B. Allotta, A. Caiti, R. Costanzi et al., "A new AUV navigation system exploiting unscented Kalman filter," Ocean Engineering, vol. 113, pp. 121-132, 2016.

[27] Y. Zhou, C. Zhang, Y. Zhang, and J. Zhang, "A new adaptive square-root unscented Kalman filter for nonlinear systems with additive noise," International Journal of Aerospace Engineering, vol. 2015, Article ID 381478, 9 pages, 2015.

[28] M. Y. Dai, H. Mu, M. Wu, and Z. Xian, "Decentralized state estimation algorithm of centralized equivalent precision for formation flying spacecrafts based on junction tree," International Journal of Aerospace Engineering, vol. 2015, Article ID 714302, 14 pages, 2015.

[29] Q. Xia, M. Rao, Y. Ying, and X. Shen, "Adaptive fading Kalman filter with an application," Automatica, vol. 30, no. 8, pp. 1333-1338, 1994.

[30] L. Ozbek and F. A. Aliev, "Adaptive fading Kalman filter with an application," Automatica, vol. 34, no. 12, pp. 1179-1182, 1998. 